\title{
Determination of the optical properties of Intralipid $20 \%$ over a broadband spectrum
}

\author{
Ali Shahin, ${ }^{* 1}$ Moustafa Sayem El-Daher, ${ }^{1}$ and Wesam Bachir ${ }^{1}$ \\ ${ }^{1}$ Biomedical Photonics Laboratory, Higher Institute for Laser Research and Applications, Damascus \\ University, 11-111 Damascus, Syria
}

Received September 06, 2018; accepted December 27, 2018; published December 31, 2018

\begin{abstract}
The aim of this study is to characterize the optical properties of Intralipid20\% using two methods: a modified KubelkaMunk model and Mie theory, and to test the applicability of a modified Kubelka-Munk model with a single integrating sphere system over a wide wavelength range $470 \div 725 \mathrm{~nm}$. Scattering coefficients estimated by these two methods were matched and the absorption effect was observed and quantified. Finally, the imaginary part of the refractive index was estimated besides scattering, absorption and anisotropy coefficients.
\end{abstract}

The tissue-simulating phantom is a virtual or synthetic tissue created to simulate the optical properties and model the transport of light in human tissue [1]. Since biological tissue is a turbid medium, constructed phantom must contain scattering and absorbing materials [2]. Fat emulsions (Intralipid, Nutralipid, Liposyn etc) are the most common materials used to simulate scattering events at both liquid and solid phantoms [3-4]. These products consist mainly of soybean oil, egg phospholipid, water and glycerin [5]. All prior researches that were aimed to characterize their optical properties showed variation in the scattering coefficient and proved the existence of an absorbing effect according to albedo variation vs wavelength [3, 6]. The variation of optical properties returned to manufacturing processes and characterization methods. Generally, all research groups regard fat emulsions as a scattering material because of their week absorption features [5, 7-8]. Because of enormous variation of normal and tumor tissue optical properties, accurate determination of phantom component optical property has a vital role.

On the other hand, there are two strategies for optical characterization. The first one is a direct method depending on a simple principle of extracting but one that requires a very thin sample to apply [9]. The second one is an indirect method which is widely used to characterize optical properties based on measuring diffuse transmission, diffuse reflection and collimated transmission, revealing the optical properties completely but using more complicated principles [10-11]. One of these analyzing principles is the Kubelka-Munk model, which is easy to use but constrained by many restrictions [12-14]. Thus, a modified Kubelka-Munk model was

\footnotetext{
*E-mail: ali.shahin88@yahoo.com
}

introduced to overcome the previous restrictions and tested over an IR region. This model takes into account a dramatic change in the refractive index between a sample and a holder by using $\boldsymbol{T}_{\boldsymbol{f}}$ Frensel power transmission coefficient, which is given by [15]:

$$
T_{f}=\frac{4 . n}{(n+1)^{2}},
$$

where $\mathrm{n}$ is the refractive index of the medium. Since a small concentration of Intralipid $20 \%$ is used, the refractive index can be considered as a refractive index of the solvent. Scattering and absorption coefficients can be retrieved by measuring diffuse transmission and reflection spectrum which are given by these correlations [15]:

$$
\begin{aligned}
& \mu_{a}=\frac{1}{d} \log \left(T_{f}^{2} / T_{d}\right)-\frac{2 \cdot R_{d} \cdot T_{f}^{2} \cdot \log \left(T_{f}^{2} / T_{d}\right)}{d \cdot\left(T_{f}^{4}-T_{d}^{2}\right)}, \\
& \mu_{s}=\frac{1}{d} \log \left(T_{d} / T_{c}\right)+\frac{2 \cdot R_{d} \cdot T_{f}^{2} \cdot \log \left(T_{f}^{2} / T_{d}\right)}{d \cdot\left(T_{f}^{4}-T_{d}^{2}\right)} .
\end{aligned}
$$

Mie theory is an analytical solution of the Maxwell's equations for the scattering of electromagnetic radiation by a single spherical particle. It provides an exact solution for the scattering and the anisotropy coefficients of perfect spheres[16]. This method requires a size distribution function, fraction and refractive index of particles besides the wavelength of radiation in the vacuum and refractive index of a solvent. Cauchy's equation was used to calculate the refractive index of water as a solvent and fat emulsion as a scattering particle [5]. Matzler algorithm was used to calculate Mie scattering parameters [17-18]. This program calculates anisotropy $g(r, \lambda)$ and scattering efficiency $Q(r, \lambda)$; given the volume of soya oil and lecithin per unit volume, which is 0.239 , the scattering coefficient and anisotropy factor for Intralipid $20 \%$ can be calculated. Determining the size of a distribution function is very complex and was not feasible in this study so we relied on Van Staveren's results [8]. Experimental determination of optical properties requires measuring diffuse transmission and reflection besides collimated transmission. Therefore, a single integrating sphere system was set up, which was composed of an integrating sphere 
(819C-IS-5.3, Newport, USA), halogen-tungsten light source (HL-2000-HP-FHSA, Ocean Optics Inc. FL), 1 mm pinhole and spectrometer (USB4000 FL, Ocean Optics Inc), as shown in Fig. 1, and collimated transmission spectroscopy which consists of a cuvette holder (CUVATT-DA, Avantes, Netherlands) as well as a spectrometer and a white light source, as shown in Fig. 2. The intralipid sample was held by a $1 \mathrm{~cm}$ path length quartz cuvette.
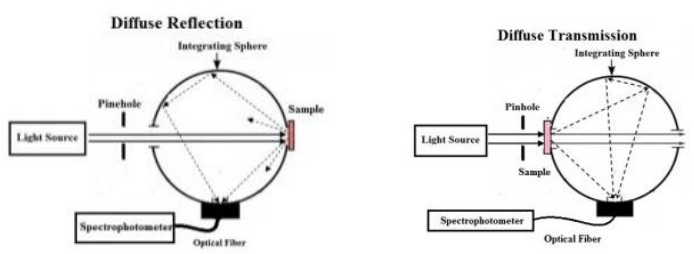

Fig. 1. Schematic diagram of the integrating sphere system.

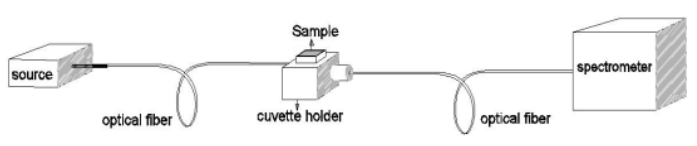

Fig. 2. Schematic diagram of collimated transmission spectroscopy.

Figure $3 \mathrm{a}$ shows the relation between the extinction coefficient and wavelength over a broad range of $470 \div 725 \mathrm{~nm}$ and the relationship of the scattering coefficient, which, calculated by the Kubelka-Munk and Mie theory with a wavelength, were shown in Fig. 3b and Fig. 3c. It can be seen that the extinction and scattering coefficients decrease exponentially as a function of wavelength over the studied wavelength range but the difference between them was in the parameters of an exponential function. The expression of an extinction coefficient in terms of wavelength was determined as follows:

$$
\mu_{t}=2.508 \cdot 10^{8} \cdot \lambda^{-2.261}
$$

For the scattering coefficient, similar results were found as follows:

$$
\begin{aligned}
& \mu_{s(\text { KM })}=2.204 \cdot 10^{8} \cdot \lambda^{-2.244}, \\
& \mu_{s \text { (Mie) }}=3.403 \cdot 10^{8} \cdot \lambda^{-2.332} .
\end{aligned}
$$

To simplify the determination of the Intralipid\%20 scattering coefficient over the studied range without using experimental technique has been determinated. The relation between $\mathrm{K}-\mathrm{M}$ and Mie scattering coefficient was estimated and given by:

$$
\mu_{s(K M)}=1.131 \cdot \mu_{s(M i e)}
$$
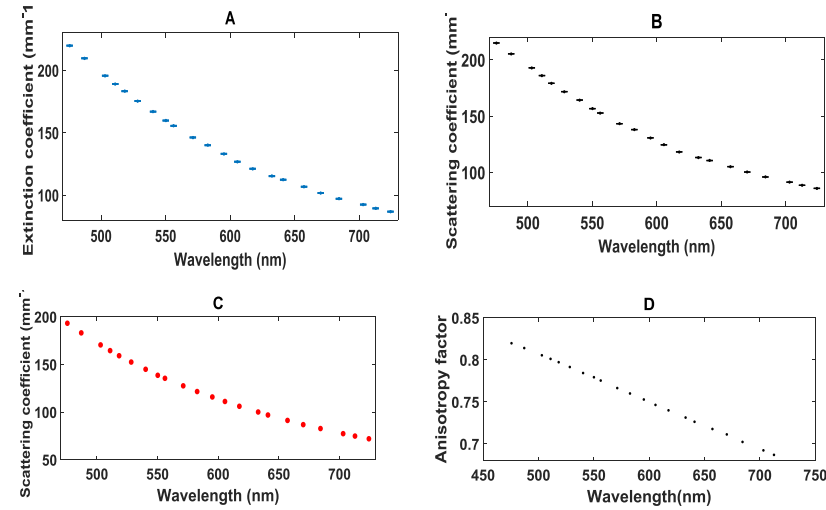

Fig. 3. a) Extinction coefficient, b) K-M scattering, c) Mie scattering and d) anisotropy vs wavelength.

Mie theory was also used to calculate the anisotropy factor over the defined wavelength range, which can be shown in (Fig. 3d). It was used to estimate reduced scattering coefficients as shown in (Fig. 4a) and (Fig. 4b) respectively. The expressions of reduced scattering coefficients calculated by Mie and K-M with a wavelength were given by:

$$
\begin{aligned}
& \mu_{s(\text { KM })}^{\prime}=7.835 \cdot 10^{3} \cdot \lambda^{-0.8597} \\
& \mu_{s(\text { Mie })}^{\prime}=13.36 \cdot 10^{3} \cdot \lambda^{-0.9636}
\end{aligned}
$$
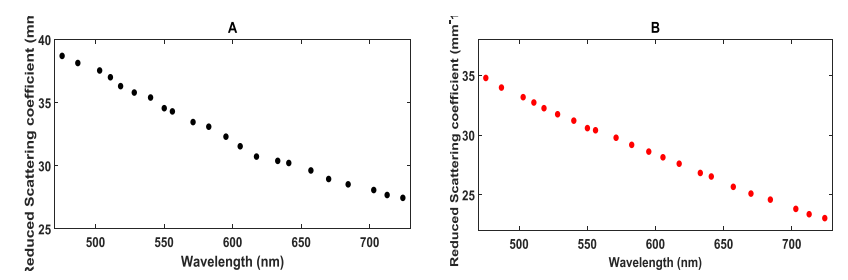

Fig. 4. a) KM reduced scattering and b) Mie reduced scattering coefficients with wavelength.

Then, albedo was calculated (Fig. 5a) and an absorption coefficient (Fig. 5b) of intralipid $20 \%$ over that range of wavelength using modified Kubelka-Munk results. The absorption coefficients that were calculated experimentally using the modified Kubelka-Munk model made the determination of an imaginary part of the refractive index of Intralipid $20 \%$ possible according to the following expression [19]:

$$
k=\mu_{a} \lambda / 4 \pi .
$$

Imaginary refractive index values were plotted vs. wavelength (Fig. 6) and correlation was estimated in the manner of Cauchy's absorbent equation, as follows:

$$
k(\lambda)=I+J / \lambda^{2}+K / \lambda^{4}
$$

where $I=-5.748 \cdot 10^{-5}, J=56.86, K=-0.9333$ for Intralipid $\% 20$ and wavelength is in nanometer. 
Figure $3 \mathrm{a}$ demonstrates the variation of an extinction coefficient with a wavelength. Obviously, the extinction coefficient decreases exponentially with wavelength and this result is confirmed by Eq. (4). Modified KubelkaMunk model and Mie theory calculations were used for two reasons: firstly, to test the modified K-M model with a single integrating sphere system over the studied range and secondly, to characterize the optical properties of Intralipid $20 \%$.
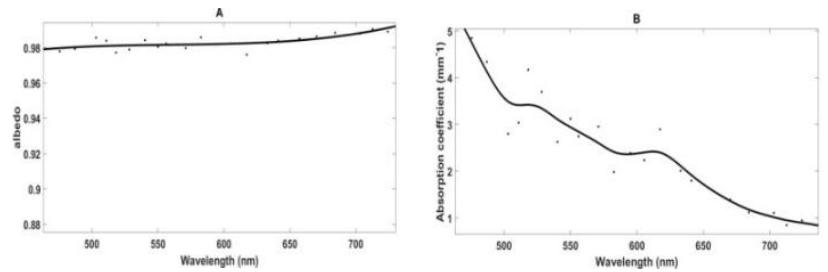

Fig. 5. a) Albedo vs wavelength, b) Absorption coefficient vs wavelength.

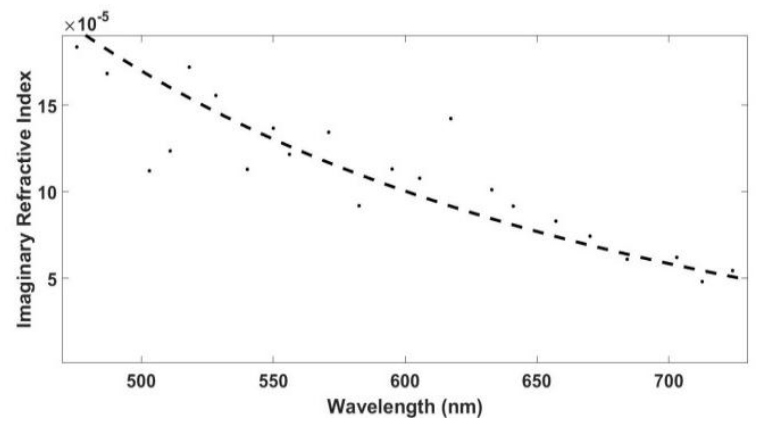

Fig.6. Imaginary refractive index as a function of wavelength.

The relation of a scattering coefficient and wavelength is exponential (Fig. 3). These values were matched and compatible with prior works $[5,7,20]$. The agreement of these values proved the validity of a modified KubelkaMunk model for the system and the applicability of Cauchy's equation to determine the real refractive index of Intralipid 20\% over the studied range. Equations (5) and (6) showed that the scattering of Intralipid 20\% is proportional to the inverse square of a wavelength which confirmed by Mie theory and this result proved that Intralipid's scattering is located between Mie and Rayleigh scatterings because of the diversity of fat emulsion particle diameters [8]. Intralipid20\% showed an absorption property because of water as a solvent but the absorption coefficient was higher than the absorption coefficient of water over the studied range, which confirmed the absorbing feature of other components [19]. In this work, absorption values varied with a wavelength and were higher than the results of previous research [20]. This can be attributed to the principle of diffusion approximation which assumed the infinitesimal values of an absorption coefficient compared to a scattering coefficient, depending on diffuse reflection only in contrast to the modified K-M model which introduced the relation of an absorption coefficient based on diffuse transmission and reflection. Additionally, Fig. 5b showed nonlinear behavior of an absorption coefficient with a wavelength. These values used to estimate the imaginary refractive index of Intralipid and its relation with a wavelength over the studied range expressed as Cauchy's equation for determining Cauchy's absorbent equation parameters. Finally, building an optical phantom precisely, requires knowledge about Intralipid20\% absorption and scattering properties. Thus, Intralipid solely based optical phantom can be used as a turbid medium for high scattering and low absorbing biological tissues. It is highly anticipated that accurate determination of a scattering coefficient of biological optical phantoms will provide more efficient Inverse Monte Carlo algorithms and, consequently, robust in-vivo estimation of real optical properties of biological tissues. This would enhance the diagnostic potential of optical instrumentation currently used for discriminating diseased biological tissues.

\section{References}

[1] B.W. Pogue, M.S. Patterson, J. Biomed. Opt. 11, 4 (2006).

[2] J. Hwang, C. Ramella-Roman, R. Nordstrom, Biomed. Opt. Expr. 3, 6 (2012).

[3] P. Ninni, F. Martelli, G. Zaccanti, Phys. Med. Biol. 56, 2 (2011).

[4] S. Flock, S. Jacques, B. Wilson, W. Star, J.C. van Gemert, Lasers Surg. Med. 4, 12 (1992).

[5] R. Michels, F. Foschum, A. Kienle, Opt. Expr. 16, 8 (2008).

[6] L. Spinelli et al., Opt. Expr. 15, 11 (2007).

[7] L. Spinelli et al., Biomed. Opt. Expr. 5, 7 (2014).

[8] H. van Staveren, C. Moes, J. van Marle, S. Prahl, J. van Gemert, Appl. Opt. 30, 31(1991).

[9] B. Wilson, M. Patterson, S. Flock, Photochem. Photobiol. 46, 5 (1987).

[10] H. Soleimanzad, H. Gurden, F. Pain, J. Biomed. Opt. 22, 1 (2017).

[11] C. Holmer et al., J. Biomed. Opt. 12, 1 (2007).

[12] S. Thennadil, OSA 25, 7 (2008).

[13]L. Yang, B. Kruse, OSA 21, 10 (2004).

[14] W. Vargas, G. Niklasson, Appl. Opt. 36, 22 (1997).

[15] A. Karinov, A. Mokeeva, E. Segeeva, P. Agrba, M. Kirillin, Opt. Spec. 115, 2 (2013).

[16] H.C. van de Hulst, Light Scattering by Small Particles (Dover Publication, New York 1981).

[17]C. Matzler, Matlab Functions for Mie Scattering and Absorption (Bern University, Bern 2002).

[18]C. Matzler, Matlab Functions for Mie Scattering and Absorption, version 2 (Bern University, Bern 2002).

[19] G. Segelstein, The complex refractive index of water [dissertation]. (University of Missouri-Kansas City, Kansas 1981).

[20] A. Shahin, W. Bachir, Pol. J. Med. Phys. Eng. 21, 4 (2017). 